\begin{tabular}{ccc}
\hline International Journal of Engineering \& Technology, 9 (3) (2020) 692-701 \\
SPC & Website: $w w w . s c i e n c e p u b c o . c o m / i n d e x . p h p / I J E T$ \\
Research paper & Technology \\
\hline
\end{tabular}

\title{
Breast cancer detection using thermal images
}

\author{
Amany M. Reda Hawas ${ }^{1 *}$, Abeer Twakol Khalil ${ }^{2}$, El Said Marzouk ${ }^{3}$ \\ ${ }^{1}$ Delta Higher Institute for Engineering and technology \\ ${ }^{2}$ Banha university of engineering \\ ${ }^{3}$ Electronics and communications Engineering, Faculty of Engineering, Mansoura University, Egypt \\ *Corresponding author E-mail: amanyhawas771@gmail.com
}

\begin{abstract}
Breast cancer is a common disease, accurate and early detection of breast cancer is very important to reduce the mortality and morbidity. Previous studies expose that thermography is a good tool for early detection of the breast cancer. In this paper, a new automatic system will be introduced for the early detection of the breast cancer using thermal images and distinguishing between normal and abnormal breasts. The proposed system is based on combining textural features and histogram of oriented gradients and bag of thermal breast images and then classifying those using three different classifiers: (i) Support vector machine; (ii) Decision tree, and k-Nearest Neighbor. This proposed system provides an automatic classification of the breast cancer using image analysis accurately in low elapsed time. Experimental results showed that cubic SVM has a maximum accuracy of $98.9 \%$, a sensitivity of $98.9 \%$, and a specificity of $99 \%$. When comparing the proposed system with the relevant systems, it's approved to be more accurate with low elapsed time in learning and testing phase that can help the clinicians in the automatic diagnosis of the breast cancer.
\end{abstract}

Keywords: Breast Cancer; Support Vector Machine; Thermal Imaging.

\section{Introduction}

Nowadays, Breast cancer records as the most diagnosed form of cancer between women, skin cancer has the first highest mortality rate and breast cancer comes second after skin cancer. Cancer is used to depict disorders related with deregulated cell growth leading to tumor formation, overrun into surrounding tissues and spread to the other parts of the body. The exact reason that caused developing in cancer have not been known however researchers have found some factors that may increase the chance of this disease such as exposure to ultraviolet radiation, smoking ,age, genatic predisposition and unhealthy life style[1] .

A normal breast have glands that connects to the surface of the skin by ducts. The glands and ducts are surrounded by connective tissue embedded in which are blood vessels, lymph nodes, lymph channels and nerves. Breast cancer can originate in any part of the breast with more than 20 types of cancer having been recognized. The most known types of breast cancer are ductal carcinoma, which originates in the ductal epithelium; and lobular carcinoma, which develops in the glands. The human body naturally manages the creation, growth, and death of the cells in their tissues.

The process starts to be abnormally when the cells are not dying at the rate they have, so the rate of cell growth will increase to be more than dying cell. Breast cancer happens when cells in the breast divide and magnify without reasonable control .Breast cancer is a wellknown disease around the world; in the USA, one in eight women will be diagnosed with breast cancer in her lifetime, and more than 40,000 is dying every year in USA[2].This number can be reduced by early detection techniques by better diagnostics, awareness campaigns, and improving detect breast cancer in the early stage when no one can feel the presence of the lump in the breast. Mammogram (low energy x-ray) used to detect breast cancer in the early stage, it is widely used but it is not perfect because it requires repeating the exposure to radiation. The women have dense tissue have high rate of false-positive results in terms with breast cancer. Ultrasound can detects tumor using sound waves passes on the surface of the tissue. MRI (magnetic resonance imaging) uses strong magnetic fields combined with pulsing radio waves to get cross section, high resolution image of the breast. Ultrasound, MRI is recommended for the positive mammogram. Excessive exposure of mammogram, ultrasound and MRI may be harmful, too costly, inaccessible or invasive methods. In this paper, the main concern is to distinguish between healthy and unhealthy breasts using thermal images that require using FLIR sc-620thermal camera which is connected to an android based smartphone.

In the proposed system we divide the paper into five sections as follows: section 1 presents introduction. Section 2 presents methods for diagnosis of breast cancer. Section 3 describes the breast thermography. In section 4 the proposed method for the segmentation of hot region based on color using k-means clustering technique. Section5 demonstrates the experimental results obtained with breast. 


\section{Thermography}

Breast thermography is an important tool in breast cancer it is considered as a diagnostic process that takes images of breasts to detect breast cancer in early stages. Breast thermography depend on the principle that the activity of chemical and blood vessel in precancerous tissue to retain existing blood vessels open and make new ones (neoangiogenesis). This process increases the temperature in the surface of the breast. Breast Thermography detect temperature change in the breast by using infrared camera and computer that analyze and produce high resolution image [3], [4].

\subsection{Data acquisition}

Thermogram is an infrared thermal image. The images are taken using FLIR SC-620 viewed in fig.1 as below, Infrared Camera having a spectral range of 7.5 to $13 \mu \mathrm{m}$ and $640 \mathrm{x} 480$ IR resolutions. The images are obtained in JPG Format. There are a lot of specific properties of this camera that should be considered before image analysis: sensitivity, temperature range, resolution, and field of view as tabulated in Table 1[5]
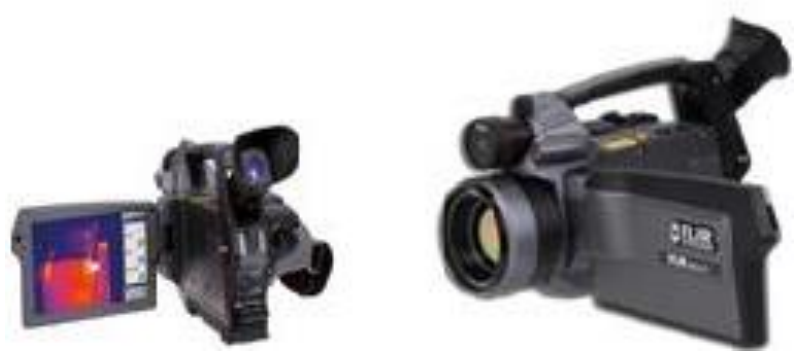

Fig. 1: FLIR SC-620.

Table 1: FLIR SC-620 Thermal Camera Specification

\begin{tabular}{lll}
\hline Camera specification & unit & Value \\
\hline Temperature Measurement Range & ${ }^{\circ} \mathrm{C}$ & {$[-40,500]$} \\
Thermal accuracy & ${ }^{\circ} \mathrm{C}$ & \pm 2 \\
Field Of View (FOV) H×V & Deg. & $24^{\circ} \times 18^{\circ}$ \\
Detector Resolution & pixel & $640 \times 480$ \\
Thermal Sensitivity & $\mathrm{mk}$ & $<40$ \\
Spectral range & $\mathrm{m} \mu$ & {$[7.5,13]$} \\
Spatial resolution & $\mathrm{mrad}$ & 0.65 \\
Minimum focus distance & $\mathrm{m}$ & 0.3 \\
\hline
\end{tabular}

\subsection{Database and data collection}

In this paper we have 60 patients, 30 of them have healthy breast and the other have unhealthy breast. Most of the patients are being classified to four categories according to the marital status (married, single), race (yellow, white, Indian, black or Mullet), and protocol and age (The age range of patients is from 21to 118 years; most of them are in the range of 50 to 70 years). Every case has provided by her personal history like,

- If there is any complains or any signs have appeared

- Family cancer.

- Family medical history.

- Last menstrual period.

- Whether or not the patient has made Mammogram, radio therapy, plastic surgery or Biopsy.

\subsection{Pre-Thermograghic imaging instruction}

Skin surface temperature can be changed by various conditions. In order to reduce error and usefulness of thermal images we should follow certain protocols to ensure that the thermal images convey accurate information as mentioned below:

- The patient must avoid prolonged sun exposure to the breast areas and chest for 5 days before the scan.

- It should be avoided to use powders, lotions, makeup, deodorants, antiperspirants, or creams on the breast on the day of the exam.

- The patient must not take a bath before 4 hours before the scan.

- It should be avoided to shave or use any types of hair removal of the breast, underarms or chest for 24 hours before the scan.

- It must avoid physical stimulation of the breasts for 24 hours before the scan.

- The patient should not do exercise for 6 hours before the scan.

- It should be avoided to take any treatment like physical therapy, acupuncture, massage, ultrasound, electrical muscle stimulation, hot or cold pack use of the back, neck, breasts or chest before 24 hours of the scan.

- The doctor should be informed if someone suffering from serious infection such as flu, or favor.

- The patient must eat light and no tea or coffee.

- The patient must stop smoking or drinking alcohol for 24 hours before the scan.

\subsection{Thermography procedure}

1) The first main rule for Infrared imaging is that it should be made in an environment that is being under full control, considering that external factors cause thermal effect. 
2) The patient should be guided to stay away from sun exposure, stimulation or treatment of Thermography the breasts, cosmetics, lotions, antiperspirants, deodorants, exercise, and bathing before the exam.

3) The temperature and humidity of imaging room have to be under control within the range of 18 to 23 Celsius, and during the imaging is in process the acceptable deviation for temperature is $1^{\circ} \mathrm{C}$.

4) The temperature range must be stable over the examination so the patient does not exposed to any shivering or perspiring.

5) The room should not contain any blast or infrared sources of heat like sunlight.

6) The floor of the room must be carpeted or the patient foot should be advised to wear his shoes to maintain neutral temperature environments.

7) The patient should go through waist-up naked acclimation over 15 min so the body reach thermal equilibrium with the environment ,in this case the variation in the temperature of the body surface occur uniformly and very slowly .at this point the thermal effects caused by outside environment or clothing are removed.

8) In the end of the acclimation period, the patient should put their hand over their head to take an afflicted image present an anatomic of the breast.

9) The infrared camera FLIR SC-620 used to capture the thermal images of the breast.

10) The infrared emanate from the skin surface convert into electrical impulses by the infrared camera.

11) The captured images are considered as graphically map of the body temperature as it visualized in color on a monitor. This image called thermograms.

12) The body surface emitted an amount of infrared radiation, the increase or the decrease of this infrared radiation indicates by the spectrum of colours. The high degree of thermal symmetry indicates to normality body and the high degree of the asymmetry indicates to abnormality.

13) Then we take the thermal images and analyse it to determine the healthy breast and unhealthy breasts like viewed in Fig. 2. below,

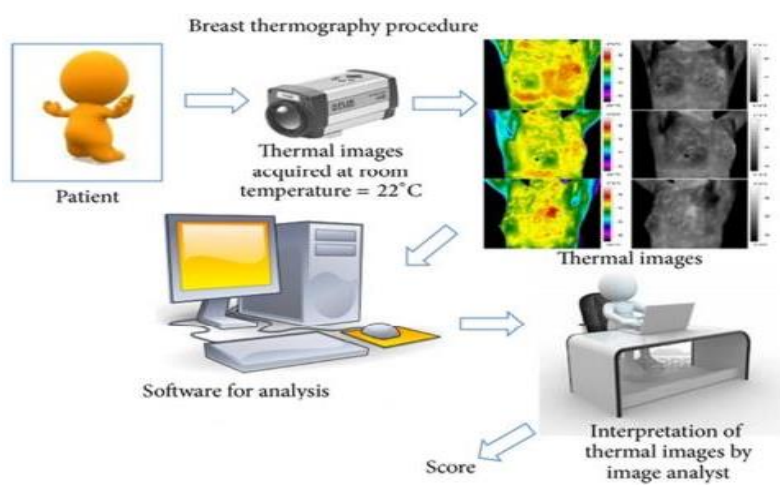

Fig. 2: Themographic Procedure.

\subsection{Sample of thermal image}

As allsurated in Fig. 3 (a) and (b) show the thermal images of Volunteers with healthy breast and unhealthy breast respectively. These thermal images are taken by following the previous instructions of Pre-Themographic Imaging.

(A)

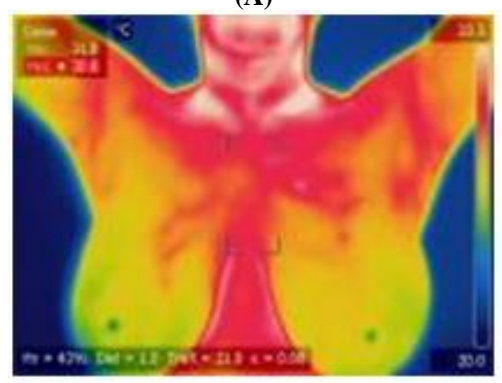

(B)

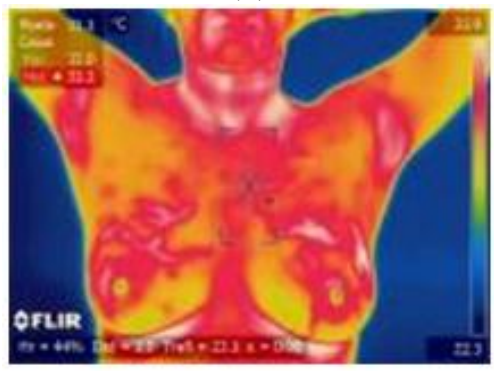

Fig. 3: A) Healthy Breast. B) Unhealthy Breast.

\section{Related work}

There are many literatures that focused on the diagnosis of breast cancer using thermal images by noticing the variation in temperature (colors), but here we mention just a few relevant papers on breast cancer detection:

Kennedy et al. at [3] declare that there is no single tool that provides perfect predictability over breast cancer detection.A combination that combines thermography with other screening techniques such as ultrasound, mammography may increase specificity and sensitivity. This paper has a result as $83 \%$ of sensitivity when using thermography alone and $95 \%$ of sensitivity when combining thermography and mammography.

Hankare et al. at [6] presented a technique that using color analysis. As declared, breast thermography detects tumors by thermal changes in the body. This paper uses K-means clustering techniques for image segmentation. They provide automated technique to detect abnormality in breast thermal images using color analysis. They stated some limitation of K-means such as unable to get the same result every time, difficulty in predicting k-values, not much efficient with global clusters.

Wahab AA et al. at[7] deals with ANN( artificial neural network) for tumor localization that uses multiple features which extracted from a series of numerical simulations. This paper presents a ANN system of 6 input, 8 neurons and 1 output with a learning rate of0.2, mo- 
mentum constant value as 0.3 and iteration rate of 20,000.this system includes various steps like data sampling then network configuration, training and optimization and finally testing and validation. This system achieved an accuracy of $96.33 \%$.

The proposed system uses active contour for segmentation, histogram of oriented gradients for feature extraction and support vector machine for classification. The system can difference between normal breast and abnormal breast and it has achieved a higher accuracy as $98.9 \%$. In table 2, it represents a comparison between the proposed system and other related work in methodology, accuracy and sensitivity.

Table 2: Related Work

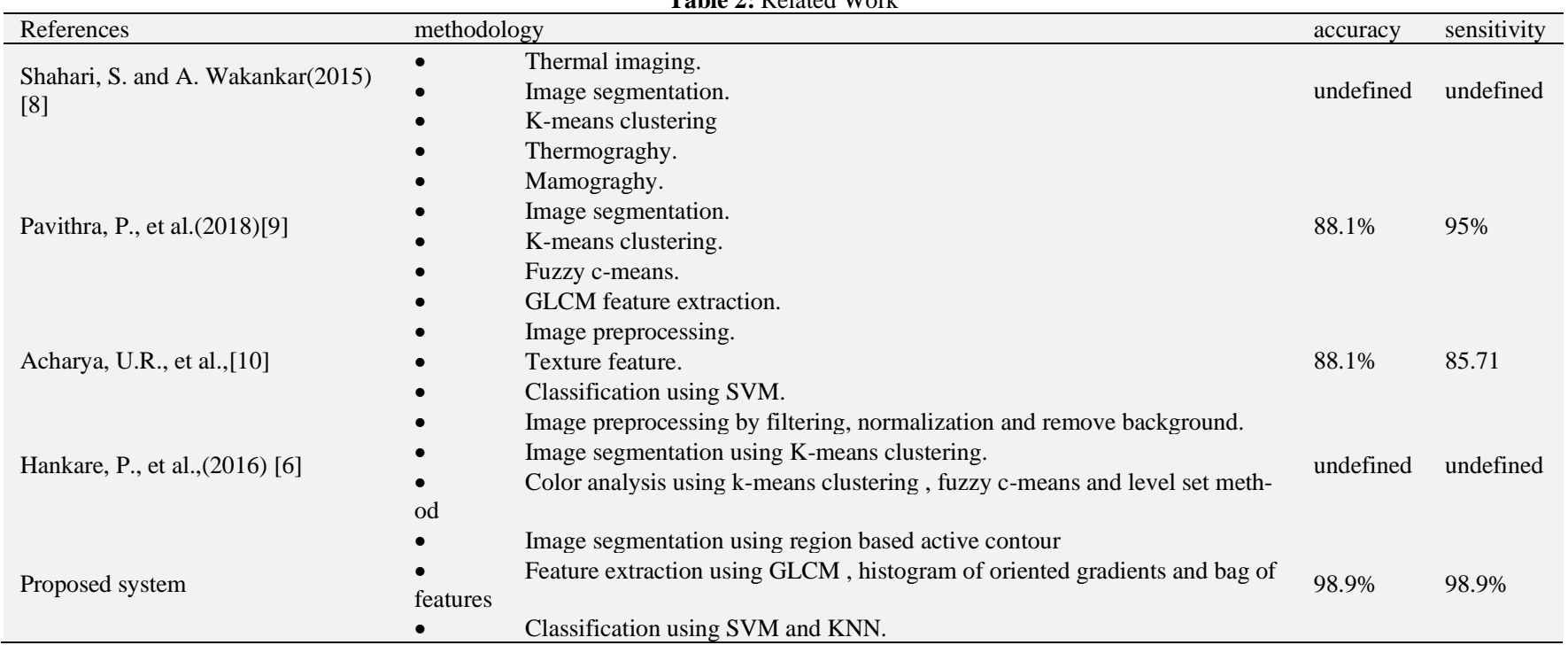

\section{Proposed method for analysis thermal images}

At this point we need to analysis thermal images so we prefer to use MATLAB image processing tool box. So we must follow some steps that showed in Fig. 4. And can describe as:

Step 1: Pre-processing to remove all unwanted data from the images like the neck.

Step 2: Segmentation to cut of the breast from the background with preservation of the size of the image.

Step 3: Select the main features of the image by feature extraction.

Step 4: Feature fusion to combine between features.

Step 5: Classification to identify each class.

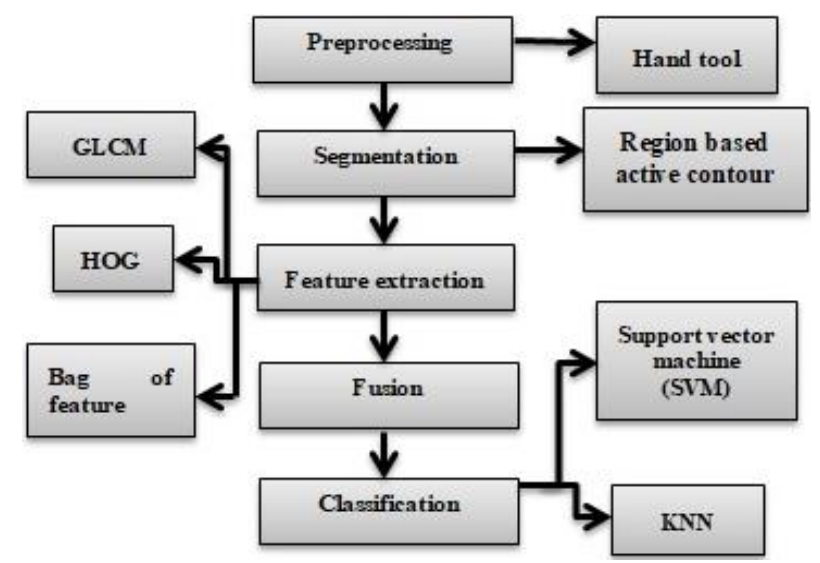

Fig. 4: The Proposed Method for Analysis Thermal Image.

\subsection{Thermal image segmentation}

Segmentation is the process that separates the required information from a data for furthermore processing. Image segmentation can be defined as the segregation of pixels of interest for effective processing [11].In this step we use region-based active contour segmentation; Active contour [12] is a segmentation technique which can be described as use of energy forces and constraints to segregate the pixels of interest from the image for furthermore processing and analysis. Contours are the boundaries that designed for the area of interest required in an image as it considered as a collection of points that undergoes interpolation process. Region-based active contours segment an image into sub regions, regions belong to only two subsets the inside and the outside of contours. Segmentation process can be summarizing in fig.5. Below; 


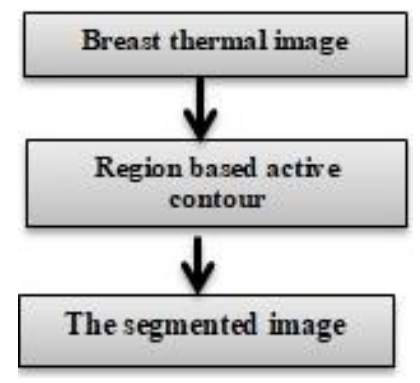

Fig. 5: Segmentation Steps.

\subsection{Feature extraction}

Feature extraction starts from a set of measured data and builds derived features prepared to be informative and non-redundant, facilitate learning and generalization steps, and in some cases it could lead to better human interpretations. The next step is feature selection; feature selection a subset of the initial features that determined from input data is suspected to be redundant so transformed into a reduced set of features. There are several methods that used for feature extraction such as transformed feature extraction (fanbeam ,principle component analysis, radon, discrete wavelet transforms[13] ,discrete cosine transform , and histogram of gradient(HOG)) ,spatial feature extraction( Harris, Surf ,fast, histogram , and textural features[14], and bag of feature) . In this paper the textural features are extracted although histogram of oriented object and bag of feature.

\subsubsection{Textural feature}

Gray level co-occurrence matrix (GLCM)is used to extract textural features , In GLCM the number of columns and rows are equal to the gray levels in the image ; let the co-occurrence matrix $\boldsymbol{p}_{\boldsymbol{i}, j}$ which represented in a square matrix with size $\mathrm{N} * \mathrm{~N}$ [14], [15],each element (i $\mathrm{j}$ ) can represent the frequency by which pixels with gray level is spatially related to pixel with gray level $\mathrm{j}$; the distance between $\mathrm{i}$ and $\mathrm{j}$ is $\mathrm{d}$,direction is $\theta$, and its probability is $\mathrm{p}(\mathrm{i}, \mathrm{j}, \mathrm{d}, \theta)$. The textural feature [15] that calculated using GLCM are contrast, energy , Correlation ,Homogeneity, Dissimilarity, Mean, Variance, Standard Deviation .Contrast is calculated by[14]

Contrast $=\sum_{i, j=0}^{L-1}(i-j)^{2} \cdot p(i, j, d, \theta)$

where, $p(I, j, d, \theta)$ is the probability value, $i$ and $j$ represent the gray intenisty,

$\mathrm{d}$ is the distance between $\mathrm{i}$ and $\mathrm{j}$, and $\theta$ is the direction.

Energy is also called angular Second Moment (ASM), it measures textural uniformity; energy will be high if the image is homogenous energy is calculated by [14],

Eeregy $=\sum_{i, j=0}^{L-1} p^{2}(i, j, d, \theta)$

Entropy is inversely proportional to energy. Entropy measures the diversity or randomness of an image. It calculated by [14],

Entropy $=\sum_{i, j=0}^{L-1} p(i, j, d, \theta) \log p(i, j, d, \theta)$

Homogeneity is also called inverse Difference Moment (IDM) is also called. Homogeneity is correlated to contrast. IDM is calculated by [14],

$I D M=\sum_{i, j=0}^{L-1} \frac{p(i, j, d, \theta)}{i+|i-j|^{2}}$

Correlation calculates the linear dependency between two pixels. Correlation is calculated by[14],

correlation $=\sum_{i, j=0}^{L-1} \frac{\left(i-\mu_{x}\right)\left(i-\mu_{y}\right) p(i, j, d, \theta)}{\sigma_{x} \sigma_{y}}$

where,

$\mu_{x}=\sum_{i, j=0}^{L-1} i \cdot p(i, j, d, \theta), \mu_{y}=\sum_{i, j=0}^{L-1} j \cdot p(i, j, d, \theta)$

$\sigma_{x}=\sum_{i, j=0}^{L-1}\left(i-\mu_{x}\right)^{2} \cdot p(i, j, d, \theta), \sigma_{y}=\left(i-\mu_{y}\right)^{2} \cdot p(i, j, d, \theta)$

Standard Deviation is calculated by [14],

$\sigma_{i}=\sqrt{\sigma_{i}^{2}}, \sigma_{j}=\sqrt{\sigma_{j}^{2}}$

Variance is calculated by[15],

$\sigma_{i}^{2}=\sum_{i, j=0}^{L-1} p_{i, j\left(i-\mu_{i}\right)^{2}}, \sigma_{j}^{2}=\sum_{i, j=0}^{L-1} p_{i, j\left(i-\mu_{j}\right)^{2}}$ 


\subsubsection{Histogram of oriented gradients (HOG)}

HOG is widely used as a feature described image region for detecting object such as human body or human face detection[16]. Color and gamma of the image should be normalized in order to increase the efficiency of the object searching. HOG is based on detector window which probes the input image region by region. As image is divided into $N \times N$ pixels; the orientation of all pixels is estimated and accumulated in an M-bins histogram of orientations. Finally, cell histograms are connected to arrange the final features vector. The steps of HOG are illustrated in fig.6 below;

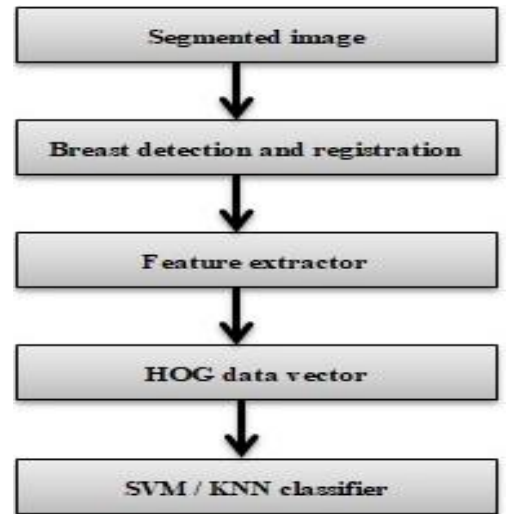

Fig. 6: Overview of the Proposed Method of Feature Extraction Using HOG.

The orientation is calculated by,

$\theta_{x, y}=\tan ^{-1} \frac{L(x, y+1)-L(x, y-1)}{L(x+1, y)-L(x-1, y)}$

where,

$\theta_{x, y}$ is the orientation of the gradient in each pixel,$L$ is the gray scale function.

\subsubsection{Bag of feature (BOF)}

BOF can be applied to image classification it treat image features as words. the BOF is applied for a set of small regions that extracted from all the image in the collection, then these small regions are presented by feature vector (descriptor), then a visual dictionary of a set of code- words is built to summarize the feature vector, finally, each image in the collection is represented by the frequency of the dictionary codewords which contains all the image in the collection represented by a codeword histogram.

The code book or visual dictionary is built using vector quantization algorithm or K-means clustering applied to the set of block descriptors that extracted from the image collection. All local features, over a training image set, are brought together independently of the source image and are clustered to learn a set of representative visual words from the whole collection. In addition, an important advantage of the BOF approach is it's not adaptation to the particular image collection to be processed. Some of these properties are used to analysis the medical image, it has been used in some problems in medical imaging [17]. The steps of BOF are illustrated in fig.7 below;

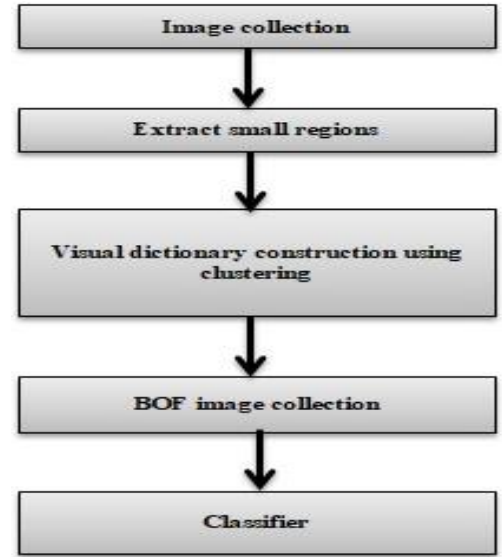

Fig. 7: Overview of the Proposed Method of Feature Extraction Using BOF.

\subsubsection{Fusion}

Image fusion is process of gathering the important information from several image into a single image. This single image is more accurate and informative than any single source image. The purpose of image fusion is not only to reduce the amount of data but also to construct images that are more understandable and appropriate for the machine and human perception. There are several methods used in feature fusion such as Principal component analysis (PCA), Discrete Cosine Transform (DCT), high pass filtering, Intensity Hue Saturation transform (IHS), Wavelet Transform (WT), Laplacian pyramids, and concatenating fusion. In this paper will be employed to combine bag of feature and histogram of oriented object to increase the accuracy of the system. 


\section{Image classification}

Classification is a two-step process, learning step and prediction step. In the learning step, the model is developed based on given training data. In the prediction step, the model is used to predict the response for given data.

\subsection{K-nearest neighbour}

In 1970's, KNN is depend on calibration dataset[14] so it has been used in statistical applications in 1970's. KNN works by comparing the test image with training images set by using a distance metric[18]. Thus, if the distance is low the image will belongs to the training set. The distance function is a basic function of $\mathrm{KNN}$; the most commonly used is Euclidean distance between two points which calculated by [18]

$\operatorname{dist}\left(X_{1}, X_{2}\right)=\sqrt{\left.\sum_{i-1}^{n} X_{1 i}-X_{2 i}\right)}$

where,

$X_{1}=\left(X_{11}, X_{12}, \ldots, X_{1 n}\right)$ and $X_{2}=\left(X_{21}, X_{22}, \ldots, X_{2 n}\right)$

The steps of KNN classification learning is summarized by (i) Calculate Similarity by using distance function; The idea to use distance measure is to find the similarity between training images and new sample (ii) find K-nearest neighbor as the smallest distance value will be ranked first and it will considered as nearest neighbor, (iii) compute the average value of the response from $\mathrm{k}$ samples then calculate the value of unknown samples. If the $\mathrm{k}$ value is a small value, the algorithm will have high variance and low. In this case the decision boundary is graphically appeared jagged. If $\mathrm{K}$ value is large the decision boundaries will graphically appeared smoother and this means that low variance and high bias. The value of $\mathrm{k}$ can be estimated by using bootstrapping procedure which depend on random sampling with replacement[19].

\subsection{Support vector machine (SVM)}

SVM is a supervised learning which uses a nonlinear mapping to transform the training data into a higher dimension. In this new dimension, SVM tries to find the linear optimal separating hyperplane. A hyperplane is a decision boundary that separates between classes. , it also find a margin to obtain the largest distance to the nearest training data [19]. The kernel function is the basis function in SVM which can transform nonlinear spaces to linear spaces. nonlinear package is represented in polynomial and sigmoid functions [20].after The user selects the suitable kernel function ,the software transforming the data and classifying it. Gaussian kernel function is calculated by [20],

$K(x, y)=\exp \left(-\gamma *(x-y)^{2}\right)$

Where, $\gamma$ is the vale of gamma

Gamma is refers to kernel scale value and it always a positive scalar value which exerted on kernel function. The separation between $\mathrm{x}$ and $\mathrm{y}$ will be large if the value of gamma is large, and if the gamma value is small the separation between $\mathrm{x}$ and $\mathrm{y}$ is small. The basic idea of support vector machine is illustrated in fig. 8. [19]There is a linear hyperplane which separates the points into two different classes, the hyperplane is a straight line in the 2- dimensional case, and there are infinitely hyperplanes that separate the training data. As illustrated in fig. 82 - hyperplane $B_{1}$ and $B_{2}$ that dividing the training data into their classes without any misclassification errors.

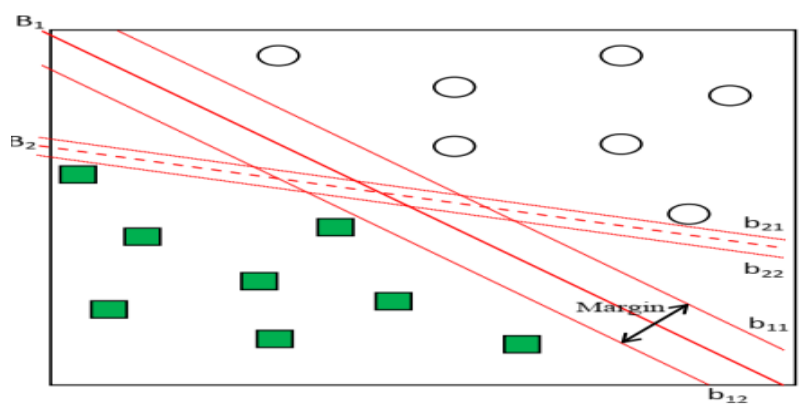

Fig. 8: An Example of 2- Classes with 2- Hyperplane.

\subsection{Decision tree (DT)}

A decision tree (DT) represented by a flowchart-like natural tree structure, where each node of this tree denotes a test sample , each branch refer to the outcome of the test, and each leaf node or terminal node refer to a class label[20]. The topmost node in the tree is considered as the root node. During tree construction, decision tree divide each node and select the best attribute that improve the accuracy of classification, many of the branches of this tree may cause outliers or noise in the training data, Tree pruning involves in removing such branches. Decision tree can be used for both regression [21] and classification kind of problem. fig. 8. shows the structure of decision tree. 


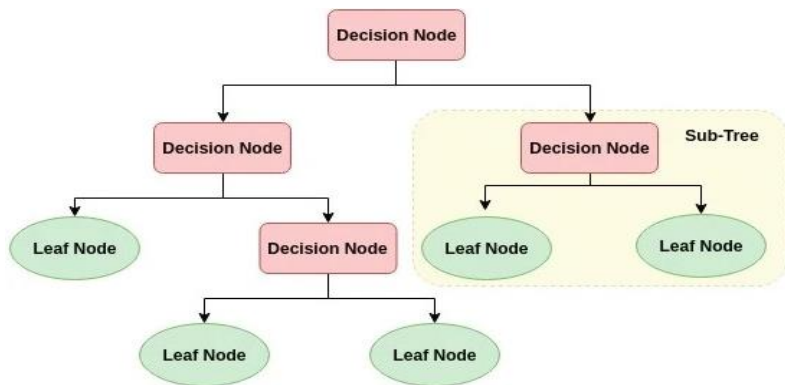

Fig. 9: The Structure of Decision Tree.

The selection of attribute measures depends on four algorithms: Gain Ratio, Chi-square, Gini Index, and Gain. Gini Index is the most common of these algorithms; the Gini Index is proportional to the homogeneity. So, if the value of Gini high, the value of homogeneity is also high that performs binary splits only. When generating decision tree, some impurities are appear in the training but the Gini index can minimize it, the Gini index is calculated by,

$\mathrm{G}\left(C \mid A_{i j}\right)=1-\sum_{k=1}^{j} p^{2}\left(C_{k} \mid A_{i j}\right)$

where, $A_{i}$ is the attribute used to branch the tree ,J is the number of classes.

\section{Results and discussion}

In the proposed system, we apply textural, histogram of oriented gradients and bag of feature to the mentioned classifiers. The largest overall accuracy is achieved from cubic SVM classifier which scored $98.9 \%$ classification accuracy. The accuracy results are shown in Fig. 9.

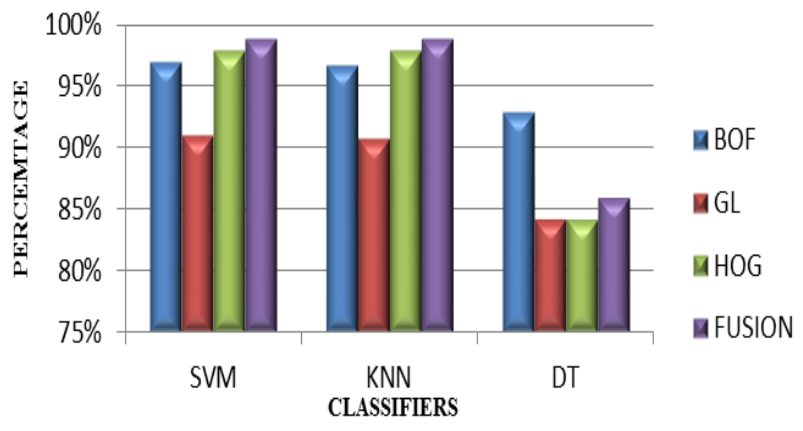

The results of accuracy showed in Fig. 9 proved that SVM on the fused feature vector between textural, histogram of oriented gradients and bag of feature outperform other classifiers. The detailed accuracy of SVM is tabulated in Table 2. Sensitivity, precision, false positive rate, and F-score are used to calculate the performance of the proposed system. They are defined as follows:

Sensitivity can be calculated by the number of correct positive predictions divided by the total number of positives. It is also called true positive rate (TPR). The best value of sensitivity is 1.0 , whereas the worst value is 0.0 , it can be calculated by [22];

$T P R=\frac{T P}{T P+F N} \times 100$

Where, TP is the true positive value and $F N$ is the false negative value.

Precision (PREC) is calculated as the number of true positive predictions divided by the total number of positive predictions. It can be also called positive predictive value (PPV). The best precision value is 1.0 , whereas the worst value is 0.0 , it can be estimated by [22];

$\mathrm{PPV}=\frac{\mathrm{TP}}{\mathrm{TP}+\mathrm{FP}} \times 100$

F-score is a harmonic mean of precision and recall, it can be estimated by [22];

$\mathrm{F}-$ score $=\frac{2 \mathrm{TP}}{2 \mathrm{TP}+\mathrm{FP}+\mathrm{FN}} \times 100$

There are important variable extracted from confusion matrix; True Negative value(TN), True Positive value (TP), False Negative value(FN), False Positive value(FP), False Predictive Rate (FPR) and False Discovery Rate (FDR) Fig.10. refers to the confusion matrix of the fused features by cubic SVM classifier. 


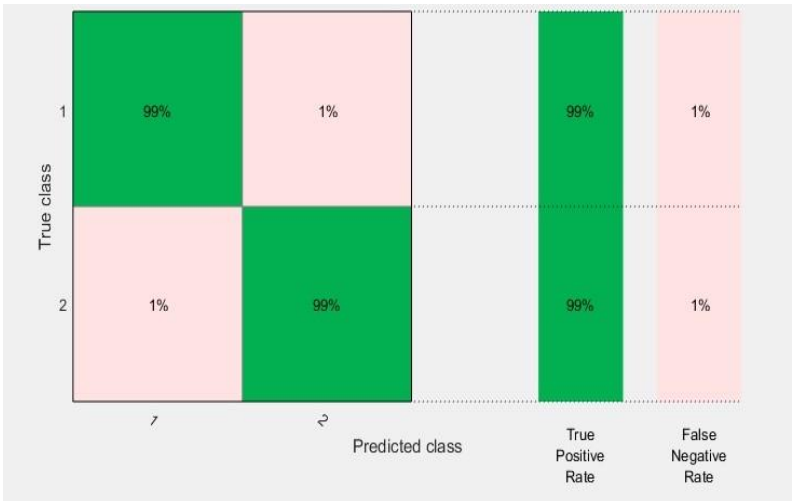

Fig. 10: Confusion Matrix (SVM).

The detailed accuracy of the fused features using cubic SVM classification, the patient classes are normal and abnormal. In Fig.10, it refers to identification of breast cancer when integrating textural, bag of features and histogram of oriented gradients to obtain whole statistical features. The value of accuracy is $98.9 \%$, sensitivity is $98.8 \%$, and false positive rate (EPR) is $1.06 \%$, precision (PREC) is 98.9 , F-score is $98.9 \%$, prediction speed is 0.097 and training time $24294 \mathrm{sec}$. When textural, bag of features and histogram of oriented gradients features have been combined, the accuracy value enhanced by $7.3 \%, 0.3 \%$ over using textural, bag of features and histogram of oriented gradients separately respectively. To measure the proposed system performance, its overall accuracy has been compared with KNN and DT. The results showed that the largest accuracy had been achieved by SVM. The classification accuracy enhanced by $0.2 \%, 12.9 \%$ over using KNN and DT respectively.

\section{Conclusions}

This paper presents a new automatic technique for diagnosis of breast cancer. Due to the rate of the spread of breast cancer around the world, especially in poor countries encourages medical organization to search for new techniques for an early and automatic diagnosis to reduce the number of mortality. It's known that traditional methods have disadvantages like it can be invasive, ionizing and compression. The latest studies discover that the thermography is a good monitoring tool of diagnosis that can see the variation in breast temperature. In the proposed system, the database of 566 thermal feet images has been acquired in special circumstances from 100 volunteers divided into two classes. Textural, histogram of oriented gradients and bag of features are extracted from the segmented breast images for further analysis. SVM, KNN, and DT are used for verifying the classification accuracy. When comparing the performance of the three classifiers, the SVM had achieved the most efficiency with maximum classification accuracy, which enhanced the classification accuracy by $7.3 \%, 0.3 \%$ over using KNN and DT respectively. The proposed system achieved a maximum classification accuracy of $98.9 \%$, a sensitivity of $98.9 \%$, and a specificity of $98.9 \%$. due to this study the medical organization can be differentiated between normal breasts and abnormal breasts accurately and can be presented in polyclinics as an aided tool to help them to take their decision about the patients.

\section{References}

[1] Doll, R. and R. Peto, The causes of cancer: quantitative estimates of avoidable risks of cancer in the United States today. JNCI: Journal of the National Cancer Institute, 1981. 66(6): p. 1192-1308. https://doi.org/10.1093/jnci/66.6.1192.

[2] Wolff, A.C., et al., Recommendations for human epidermal growth factor receptor 2 testing in breast cancer: American Society of Clinical Oncology/College of American Pathologists clinical practice guideline update. Archives of Pathology and Laboratory Medicine, 2014. 138(2): p. 241-256. https://doi.org/10.5858/arpa.2013-0953-SA.

[3] Kennedy, D.A., T. Lee, and D. Seely, A comparative review of thermography as a breast cancer screening technique. Integrative cancer therapies, 2009. 8(1): p. 9-16. https://doi.org/10.1177/1534735408326171.

[4] Ring, E. and K. Ammer, The technique of infrared imaging in medicine. Thermology international, 2000. 10(1): p. 7-14.

[5] Korkolainen, P., Lämmön regeneroinnin hyödyntäminen hydraulipaineakussa. 2014.

[6] Hankare, P., et al., Breast cancer detection using thermography. Int. Res. J. Eng. Technol, 2016. 4(3): p. 2395-2356.

[7] Wahab, A.A., et al. Tumor localization in breast thermography with various tissue compositions by using Artificial Neural Network. in 2015 IEEE Student Conference on Research and Development (SCOReD). 2015. IEEE. https://doi.org/10.1109/SCORED.2015.7449383.

[8] Shahari, S. and A. Wakankar. Color analysis of thermograms for breast cancer detection. in 2015 International Conference on Industrial Instrumentation and Control (ICIC). 2015. IEEE. https://doi.org/10.1109/IIC.2015.7151001.

[9] Pavithra, P., et al., The effect of thermography on breast cancer detection. Systematic Reviews in Pharmacy, 2018. 9(1): p. 10-16. https://doi.org/10.5530/srp.2018.1.3.

[10] Acharya, U.R., et al., Thermography based breast cancer detection using texture features and support vector machine. Journal of medical systems, 2012. 36(3): p. 1503-1510. https://doi.org/10.1007/s10916-010-9611-z.

[11] Duarte, A., et al., Segmentation algorithms for thermal images. Procedia Technology, 2014. 16: p. 1560-1569. https://doi.org/10.1016/j.protcy.2014.10.178.

[12] Baswaraj, D., A. Govardhan, and P. Premchand, Active contours and image segmentation: The current state of the art. Global Journal of Computer Science and Technology, 2012.

[13] Ghazali, K.H., et al. Feature extraction technique using discrete wavelet transform for image classification. in 2007 5th Student Conference on Research and Development. 2007. IEEE. https://doi.org/10.1109/SCORED.2007.4451366.

[14] Minarno, A.E., et al. Texture feature extraction using co-occurrence matrices of sub-band image for batik image classification. in 2014 2nd International Conference on Information and Communication Technology (ICoICT). 2014. IEEE. https://doi.org/10.1109/ICoICT.2014.6914074.

[15] Shijin, K. and V. Dharun, Extraction of texture features using GLCM and shape features using connected regions. International Journal of Engineering \& Technology, 2016. 8(6): p. 2926-2930. https://doi.org/10.21817/ijet/2016/v8i6/160806254.

[16] Carcagnì, P., et al., Facial expression recognition and histograms of oriented gradients: a comprehensive study. SpringerPlus, 2015. 4(1): p. 645. https://doi.org/10.1186/s40064-015-1427-3.

[17] Cruz-Roa, A., J.C. Caicedo, and F.A. González, Visual pattern mining in histology image collections using bag of features. Artificial intelligence in medicine, 2011. 52(2): p. 91-106. https://doi.org/10.1016/j.artmed.2011.04.010. 
[18] Thanh Noi, P. and M. Kappas, Comparison of random forest, k-nearest neighbor, and support vector machine classifiers for land cover classification using Sentinel-2 imagery. Sensors, 2018. 18(1): p. 18. https://doi.org/10.3390/s18010018.

[19] Entezari-Maleki, R., A. Rezaei, and B. Minaei-Bidgoli, Comparison of classification methods based on the type of attributes and sample size. Journal of Convergence Information Technology, 2009. 4(3): p. 94-102. https://doi.org/10.4156/jcit.vol4.issue3.14.

[20] Carugo, O., F. Eisenhaber, and Carugo, Data mining techniques for the life sciences. 2016: Springer. https://doi.org/10.1007/978-1-4939-3572-7.

[21] Raschka, S., Python machine learning. 2015: Packt Publishing Ltd.

[22] Hossin, M. and M. Sulaiman, A review on evaluation metrics for data classification evaluations. International Journal of Data Mining \& Knowledge Management Process, 2015. 5(2): p. 1. https://doi.org/10.5121/ijdkp.2015.5201. 